\title{
Sinus fungal balls: characteristics and management in patients with host factors for invasive infection*
}

\author{
Guillaume Toussain', Françoise Botterel2,3,4, Issam Abd Alsamad", \\ Françoise Zerah-Lancner ${ }^{6}$, Virginie Prulière-Escabasse ${ }^{1,4,7,8,}$, \\ André Coste ${ }^{1,4,7,8}$, Jean F. Papon $1,4,7,8,9$ \\ Hopital intercommunal, service d'ORL et de chirurgie cervico-faciale, Creteil, F-94010, France \\ Service de Parasitologie-Mycologie, Hôpital Henri Mondor, (AP-HP) Créteil, France \\ UMR BIPAR, -UPEC, Creteil, F-94010, France \\ Universite Paris Est Creteil, Faculte de Medecine, UMR_S841, Creteil, F-94010, France \\ Hopital intercommunal, service d'anatomo-pathologie (laboratoire de microscopie electronique), Creteil, F-94010, France \\ AP-HP, Groupe hospitalier Henri-Mondor - Albert Chenevier, service de physiologie et d'explorations fonctionnelles, Creteil, \\ F-94010, France \\ AP-HP, Groupe hospitalier Henri-Mondor - Albert Chenevier, service d'ORL et de chirurgie cervico-faciale, Creteil, F-94010, France \\ INSERM, U955, Creteil, F-94010, France \\ INSERM, U933 Paris, F-75012, France
}

Rhinology 50: 269-276, 2012

DOI:10.4193/Rhino11.223

*Received for publication:

October 20, 2011

Accepted: May 19, 2012

\section{Abstract}

Background: The characteristics of sinus fungal ball (SFB), classically considered being a non-invasive form of fungal infection, in patients with host factors for invasive fungal infection (IFI) are unknown.

Objective: To characterize SFB and their management in patients with host factors for IFI.

Methodology: Retrospective single-centre study of the clinical, radiology, histology and mycology records of patients treated for SFB between 1997 and 2007. Patients with and without host factors for IFI were compared.

Results: One hundred eighty one patients were classified into two groups: 19 (group 1) with and 162 (group 2) without host factors for IFI. In group 1, SFB were asymptomatic in $26.3 \%$ of the cases, ethmoido-sphenoidal sinuses were more frequently involved than in group 2 and fungal culture was positive in $37.5 \%$ of the cases. The main species was Aspergillus sp. in both groups. Four cases of complicated SFB were observed, only in patients of group 1. Cure without recurrence was obtained in both groups by endonasal surgery, combined with triazole therapy in complicated forms with osteolysis.

Conclusion: In patients with host factors for IFI, SFB more frequently involves deep sinuses and can be complicated by clinical signs suggestive of invasion and radiological signs of osteolysis, with no histological evidence of fungal invasion.

Key words: sinus fungal ball, fungal infection, sinusitis, endoscopic surgery, histopathology 


\section{Introduction}

Sinus fungus ball (SFB) was first described by Plaignaud at the end of the 18th century ${ }^{(1)}$. It usually consists of localized non-invasive sinusitis, mainly involving the maxillary sinus ${ }^{(2)}$. The pathophysiology of SFB remains unclear, but some authors have highlighted the potential role of root canal cement in the pathogenesis of SFB ${ }^{(3-7)}$. Once the diagnosis of SFB has been established, curative treatment is clearly defined and is based on complete surgical removal of SFB without the need for antifungal therapy ${ }^{(8)}$.

Only a few clinical case reports of complicated SFB have been reported ${ }^{(9-11)}$, especially in immunocompromised patients, suggesting that the outcome of SFB could be influenced by host factors. Various host factors, predisposing to invasive fungal infections (IFI) have been described in the literature: neutrophil dysfunction and neutropenia, uncontrolled diabetes mellitus, hemochromatosis, previous organ or allogeneic stem cell transplants, prolonged use of corticosteroids at a mean minimum dose of $0.3 \mathrm{mg} / \mathrm{kg} /$ day of prednisone equivalent for 13 weeks, treatment with other recognized T cell immunosuppressants, and inherited severe immunodeficiency ${ }^{(2,12)}$. However, a few studies on large cohorts of patients with SFB have been published ${ }^{(8,13,14)}$ but none of them included specific analysis of patients with SFB and host factors for IFI.

The aim of this study was to analyse a large series of patients with SFB and to compare the characteristics of SFB in patients with and without host factors for IFI.

\section{Patients and methods}

Patients

The medical records of patients operated in our hospital between January 1st 1997 and December 31st 2007 for caseous sinusitis (either fungal or bacterial) were reviewed. The medical records of patients operated for sinus neoplasia and nasal polyposis were excluded from the initial selection. The study population consisted of patients with a final diagnosis of SFB, based on histopathological examination showing a matted, dense conglomeration of hyphae, as previously reported ${ }^{(15)}$. All other types of caseous sinusitis operated during the same period were excluded from the study when histopathological criteria of SFB were absent. Patients were classified into group 1 or 2 according to the presence or absence of at least one host factor for IFI, respectively.

The following preoperative characteristics were assessed in the two groups: age, gender, nasal, neurological and ophthalmological symptoms, radiological features (sinus localisation, radiological characteristics, sinus bone alteration, perisinus extension), white blood count, histopathology, mycology, surgical and medical treatments, and outcome. Institutional review board (CPP-Ile de France IX) approval was obtained for this research that involved data collection with no intervention.

\section{Laboratory investigations}

Histopathology: Histological examination using conventional Hematoxylin-Eosin-Saffron (HES) and Gomori-Grocott and Periodic Acid Schiff (PAS) stains was systematically performed. In the presence of imaging signs of extension beyond the sinuses or osteolysis, the surgeon systematically performed sinus mucosa biopsy that was also examined to detect the presence of invasive sinusitis.

Mycology: Samples of SFB sent to the microbiology laboratory were submitted to direct examination with optical brighteners. After grinding, samples were then seeded onto Sabouraud agar slopes with antibiotics and incubated for 21 days at $30^{\circ} \mathrm{C}$. Fungus identification of positive cultures was based on phenotypic features with macroscopic and microscopic examination.

\section{Statistical analyses}

All analyses were performed using a statistical software package (Statview 4; SAS Institute, Grenoble, France). An unpaired t-test was used for continuous variables and a chi-square test was used for categorical variables. When necessary, Yates' correction or Fisher's exact test were used. A p value $<0.05$ was considered significant.

\section{Results}

Patient characteristics

Between 1997 and 2007, 605 patients were operated for caseous sinusitis in our department; 181 (30\%) of these patients had a histological diagnosis of SFB. The mean age of this population was $53.7 \pm 14.6$ years (range: 29 - 86 years), and women were significantly older ( $55.2 \pm 14.3$ years) than men ( $50.5 \pm$ 14.5 years) $(p=0.04)$. The sex ratio was 2.17 (F/M: 124/57) and the female predominance persisted regardless of the sinus involved. Nineteen patients (10.5\%) presented at least one host factor for IFI, defined as group 1, and 162 patients had no host factors for IFI (89.5\%), defined as group 2. The host factors for IFI identified in group 1 were: uncontrolled diabetes ( $n=$ 9), prolonged use of systemic corticosteroids ( $n=3)$, kidney transplant $(n=1)$, haematologic malignancies $(n=2)$, treatment with T cell immunosuppressants for solid cancers $(n=2)$ and severe immunodeficiency secondary to HIV infection ( $\mathrm{n}=$ 2) (Table 2)

No significant difference was observed between the two groups for age, sex ratio, symptoms and white blood count (Table 1). Nineteen patients (10.5\%) had no symptoms on diagnosis, $26.3 \%$ in group 1 vs $11.7 \%$ in group 2, but this difference was not statistically significant $(p=0.14)$. When present, symptoms consisted of (in decreasing order of frequency): rhinorrhea, craniofacial pain, nasal obstruction and cacosmia (Table 1). Acute complicated forms were observed in four patients, only in group 1: two cases of isolated external 
Table 1. Comparison of clinical and sinus CT features of sinus fungus balls between patients with or without host factors for invasive fungal infection (group 1 and group 2, respectively).

\begin{tabular}{|c|c|c|c|}
\hline & Group $1(n=19)$ & Group $2(n=162)$ & $\mathbf{p}$ \\
\hline Age $($ mean \pm SD) & $59.9 \pm 14.8$ & $53 \pm 14.3$ & ns \\
\hline Sex ratio (F/M) & 2.8 & 2.11 & ns \\
\hline \multicolumn{4}{|l|}{ Clinical symptoms } \\
\hline Rhinorrhea (\%) & $10(52.6)$ & $118(72.8)$ & ns \\
\hline Craniofacial pain (\%) & $9(47.3)$ & $78(48.1)$ & ns \\
\hline Nasal obstruction (\%) & $5(26.3)$ & $40(24.7)$ & ns \\
\hline Cacosmia (\%) & $3(15.8)$ & $19(11.7)$ & ns \\
\hline \multicolumn{4}{|l|}{ White blood count } \\
\hline Total $($ mean \pm SD) & $7837 \pm 2667$ & $7095 \pm 1696$ & ns \\
\hline Neutrophils (mean \pm SD) & $4916 \pm 1826$ & $4292 \pm 1610$ & ns \\
\hline \multicolumn{4}{|l|}{ SFB site } \\
\hline Maxillary (\%) & $11(57.9)$ & $137(84.6)$ & 0.03 \\
\hline Sphenoidal or ethmoidal (\%) & $6(31.6)$ & $21(13)$ & 0.03 \\
\hline Frontal (\%) & 0 & 0 & $\mathrm{nr}$ \\
\hline Bifocal (\%) & $2(10.5)$ & $4(2.5)$ & ns \\
\hline \multicolumn{4}{|l|}{ CT signs } \\
\hline Partial opacity (\%) & $2(10.5)$ & $42(25.9)$ & ns \\
\hline Bone thickening (\%) & $12(63)$ & $114(70.3)$ & ns \\
\hline Meatus enlargement (\%) & $2(10.5)$ & $9(5.9)$ & ns \\
\hline
\end{tabular}

SFB: sinus fungus ball; CT: computed tomography; ns: not significant; nr: not relevant. An unpaired t-test was used for continuous variables and a chi-square test was used for categorical variables. When necessary, Yates' correction or Fisher's exact test were used. A p-value $<0.05$ was considered significant.

ophthalmoplegia, one case of meningeal syndrome and one case of external ophthalmoplegia associated with meningeal syndrome (Table 2). In three of these four cases, the patients were asymptomatic before onset of the complication.

High-resolution CT was available for all patients. In the four acute complicated forms, contrast-enhanced CT was also available, as well as MRI for two patients. CT showed predominant maxillary sinus involvement followed by sphenoid sinus involvement (Table 1). No frontal sinus involvement was observed in this population. Bifocal sites (bilateral maxillary, ethmoidosphenoidal or ethmoido-maxillary sinuses) were observed in two patients in group 1 and in four patients in group 2. The SFB was identified as a heterogeneous opacity partially $(24.3 \%)$ or completely (75.7\%) filling the sinus, sometimes associated with an iron-like density (58.2\%) with or without microcalcifications. The bony wall of the sinus was thickened in $69.6 \%$ of cases and the middle meatus or sphenoidal ostium was enlarged in $6.0 \%$ of cases. No significant difference was observed between the two groups in terms of CT signs (Table 1). The maxillary sinus was affected significantly less frequently in group 1 than in group $2(p=0.03)$, while sphenoidal and ethmoidal sinuses were involved significantly more frequently in group $1(p=$ 0.03). In the four acute complicated forms, the SFB was exclusively localized in the sphenoid sinus and contrast-enhanced CT and/or MRI showed one or more areas of osteolysis of the sinus walls in three cases associated with perisinus contrast enhancement in two cases (Figure 1). No signs of cerebral or intra-orbital collection were present in these cases.

\section{Laboratory investigations}

\section{Histopathology}

Conventional HES, Gomori-Grocott and PAS stains were available for all patients and revealed matted, dense conglomerations of hyphae. In the four acute complicated forms, biopsy of the sinus mucosa was performed and no cases of invasion by the fungal agent or allergic mucin or granulomatous inflammatory response were detected. 
Table 2. Clinical, radiological and mycological features of sinus fungus balls in patients with host factors for invasive fungal infection (group 1).

\begin{tabular}{|c|c|c|c|c|c|}
\hline Patient & Host factors for IFI & Symptoms & Radiological signs & Sinus & Fungus culture \\
\hline 1 & $\begin{array}{l}\text { Cyclophosphamide (lgG } \\
\text { myeloma) }\end{array}$ & $\begin{array}{l}\text { Rhinorrhea } \\
\text { Nasal obstruction }\end{array}$ & $\begin{array}{l}\text { Complete heterogeneous opacity } \\
\text { Iron-like density with microcalcifications } \\
\text { Bone thickening }\end{array}$ & M & A. versicolor \\
\hline 2 & Type II diabetes (HbA1c 8.1) & $\begin{array}{l}\text { Rhinorrhea } \\
\text { Nasal obstruction } \\
\text { Craniofacial pain }\end{array}$ & $\begin{array}{l}\text { Complete heterogeneous opacity } \\
\text { Bone thickening }\end{array}$ & $S$ & Negative \\
\hline 3 & $\begin{array}{l}\text { Prednisolone } 5 \mathrm{mg} / \mathrm{d}> \\
24 \text { months (rheumatoid } \\
\text { arthritis) }\end{array}$ & $\begin{array}{l}\text { Rhinorrhea } \\
\text { Craniofacial pain }\end{array}$ & $\begin{array}{l}\text { Complete heterogeneous opacity } \\
\text { Iron-like density with microcalcifications } \\
\text { Bone thickening }\end{array}$ & $\mathrm{S}$ & nd \\
\hline 4 & Type II diabetes (HbA1c 6.1) & Asymptomatic & $\begin{array}{l}\text { Complete heterogeneous opacity Mu- } \\
\text { cocele }\end{array}$ & $E$ & Negative \\
\hline 5 & $\begin{array}{l}\text { Infliximab + Prednisolone } 5 \\
\text { mg/d > } 24 \text { months (ankylos- } \\
\text { ing spondylitis) }\end{array}$ & $\begin{array}{l}\text { Rhinorrhea } \\
\text { Craniofacial pain }\end{array}$ & $\begin{array}{l}\text { Partial heterogeneous opacity } \\
\text { Iron-like density with microcalcifications } \\
\text { Bone thickening }\end{array}$ & M & Negative \\
\hline 6 & Type II diabetes (HbA1c 6.8) & $\begin{array}{l}\text { Rhinorrhea } \\
\text { Nasal obstruction } \\
\text { Craniofacial pain }\end{array}$ & $\begin{array}{l}\text { Complete heterogeneous opacity } \\
\text { Iron-like density with microcalcifications } \\
\text { Bone thickening } \\
\text { Middle meatus enlargement }\end{array}$ & M & Negative \\
\hline 7 & $\begin{array}{l}\text { Rituximab (Lymphoplasma- } \\
\text { cytic hematologic malig- } \\
\text { nancy) }\end{array}$ & Asymptomatic & $\begin{array}{l}\text { Complete heterogeneous opacity } \\
\text { Bone thickening }\end{array}$ & M & A. fumigatus \\
\hline 8 & $\begin{array}{l}\text { Imatinib (Gastrointestinal } \\
\text { stromal tumor) }\end{array}$ & Asymptomatic & Complete heterogeneous opacity & M & nd \\
\hline 9 & Type II diabetes (HbA1c 7.4) & $\begin{array}{l}\text { Rhinorrhea } \\
\text { Craniofacial pain }\end{array}$ & $\begin{array}{l}\text { Complete heterogeneous opacity } \\
\text { Iron-like density with microcalcifications } \\
\text { Bone thickening }\end{array}$ & M & Negative \\
\hline 10 & $\begin{array}{l}\text { Prednisolone } 5 \mathrm{mg} / \mathrm{d}+\text { myco- } \\
\text { phenolate mofetil } 500 \mathrm{mg} / \mathrm{d} \\
+ \text { tacrolimus } 2.5 \mathrm{mg} / \mathrm{d}>24 \\
\text { months (kidney transplanta- } \\
\text { tion) }\end{array}$ & $\begin{array}{l}\text { Rhinorrhea } \\
\text { Cacosmia }\end{array}$ & $\begin{array}{l}\text { Partial heterogeneous opacity } \\
\text { Iron-like density with microcalcifications } \\
\text { Bone thickening }\end{array}$ & M & A. fumigatus \\
\hline 11 & $\begin{array}{l}\text { HIV infection }(C D 4+=150 / \\
\text { mm3) }\end{array}$ & Asymptomatic & $\begin{array}{l}\text { Complete heterogeneous opacity } \\
\text { Bone thickening }\end{array}$ & $\mathrm{S}$ & Negative \\
\hline 12 & Type II diabetes (HbA1c 9.4) & $\begin{array}{l}\text { Rhinorrhea } \\
\text { Craniofacial pain } \\
\text { Cacosmia }\end{array}$ & $\begin{array}{l}\text { Complete heterogeneous opacity } \\
\text { Iron-like density with microcalcifications } \\
\text { Middle meatus enlargement }\end{array}$ & M & nd \\
\hline 13 & $\begin{array}{l}\text { Prednisolone } 7 \mathrm{mg} / \mathrm{d}> \\
24 \text { months (rheumatoid } \\
\text { arthritis) }\end{array}$ & Asymptomatic & Complete heterogeneous opacity & M & Negative \\
\hline 14 & Type II diabetes (HbA1c 7.7) & $\begin{array}{l}\text { Rhinorrhea Nasal obstruc- } \\
\text { tion Craniofacial pain } \\
\text { Cacosmia }\end{array}$ & $\begin{array}{l}\text { Complete heterogeneous opacity } \\
\text { Iron-like density with microcalcifications } \\
\text { Bone thickening }\end{array}$ & M & Negative \\
\hline 15 & Type II diabetes (HbA1c 7) & $\begin{array}{l}\text { Rhinorrhea } \\
\text { Nasal obstruction } \\
\text { Craniofacial pain }\end{array}$ & $\begin{array}{l}\text { Complete heterogeneous opacity } \\
\text { Iron-like density with microcalcifications } \\
\text { Bone thickening }\end{array}$ & M & Negative \\
\hline 16 & Type II diabetes (HbA1c 7.4) & External ophthalmoplegia & $\begin{array}{l}\text { Complete heterogeneous opacity } \\
\text { Bone thickening }\end{array}$ & $M+S$ & nd \\
\hline 17 & $\begin{array}{l}\text { Paclitaxel }+ \text { Navelbine }+\mathrm{Cis} \\
\text { Platinum (solid cancer with } \\
\text { multiple metastases) }\end{array}$ & $\begin{array}{l}\text { Craniofacial pain } \\
\text { External ophthalmoplegia }\end{array}$ & $\begin{array}{l}\text { Complete heterogeneous opacity } \\
\text { Iron-like density with microcalcifications } \\
\text { Osteolysis of the sinus } \\
\text { Perisinus contrast enhancement }\end{array}$ & $\mathrm{S}$ & A. versicolor \\
\hline 18 & Type I diabetes (HbA1c 7.8) & $\begin{array}{l}\text { External ophthalmoplegia } \\
\text { Meningeal syndrome }\end{array}$ & $\begin{array}{l}\text { Complete heterogeneous opacity } \\
\text { Sinus parietal osteolysis } \\
\text { Perisinus contrast enhancement }\end{array}$ & $S$ & S. commune \\
\hline 19 & HIV infection (CD4+ 50/mm3) & Meningeal syndrome & $\begin{array}{l}\text { Complete heterogeneous opacity } \\
\text { Iron-like density without microcalcifica- } \\
\text { tions. Osteolysis of the sinus }\end{array}$ & $\mathrm{E}+\mathrm{S}$ & C. glabrata \\
\hline
\end{tabular}




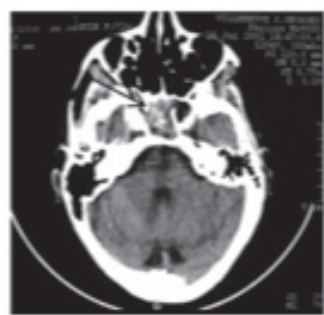

A

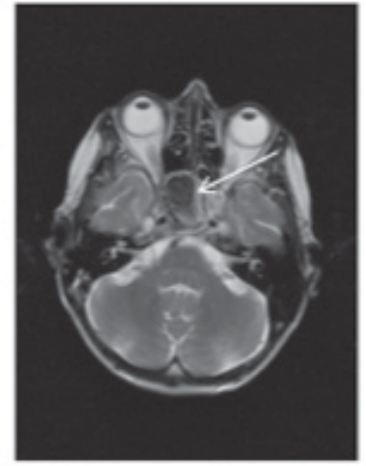

C

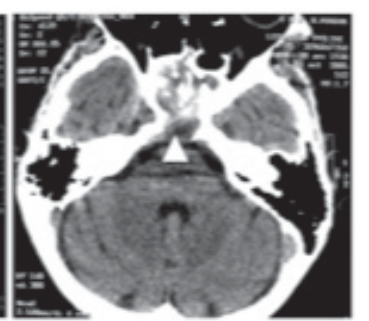

B

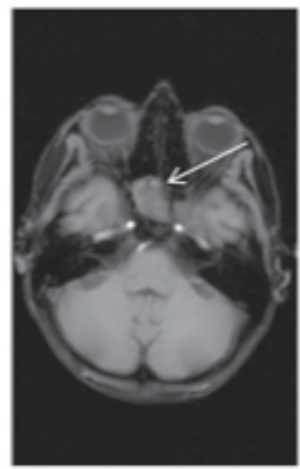

D
Figure 1. Computed tomography (A, B) and magnetic resonance imaging (C, D) of a sphenoidal fungus ball (patient 17 , group 1). Complete heterogeneous opacity of the right sphenoid sinus identified in 2002 (A, black arrow), not treated. In 2005, acute complication (external ophthalmoplegia) revealing the same opacity of the right sphenoid sinus and osteolysis of the posterior wall of the sinus ( $B$, arrowhead). Magnetic resonance imaging eliminates intracranial abscess and shows the fungus ball as a low-intensity image on T2-weighted images (C) with a heterogeneous signal on T1-weighted images (D) (white arrows).

\section{Mycology}

Mycological investigations were performed on 119 samples (65.7\%): 16/19 (84.0\%) in group 1 and 103/162 (63.5\%) in group 2. Direct examination showed thin, regular and parallel hyphae in 98 cases (82.3\%): 16/16 (100\%) in group 1 and $82 / 103(79.6 \%)$ in group 2. Cultures were positive in 37 cases (31\%): 6/16 (37.5\%) in group 1 and 31/103 (30.0\%) in group 2 (Table 3). All patients with positive cultures also had a positive direct examination. The main species was Aspergillus sp. (81\%) found in 4 patients in group 1 and 26 patients in group 2 .

Aspergillus sp. was isolated less frequently in group 1, but the difference between the two groups was not statistically significant. Other fungi identified were Candida $\mathrm{sp}(\mathrm{n}=3)$, Schizophyllum commune $(\mathrm{n}=1)$, Mucor $\mathrm{sp}(\mathrm{n}=2)$ and Cladosporium sp. $(n=1)$. In the two cases with meningeal syndrome, fungal and bacterial cultures of cerebrospinal fluid were sterile.

\section{Treatment and outcome}

SFB were removed via an exclusively endoscopic approach under general anaesthesia in all patients. Antifungal therapy was administered to the 3 acute complicated cases with osteolysis of the sinus wall. This treatment consisted of triazoles (voriconazole $400 \mathrm{mg} / \mathrm{d}$ in two cases and itraconazole $400 \mathrm{mg} / \mathrm{d}$ in one case), initiated immediately after surgery, without associated antibiotic therapy. These treatments were administered for at least three months with a regular dosage for the itraconazole regimen. After a mean follow-up of 10.2 months (range: 6 to
72 months), no SFB-related death and no SFB recurrence were observed. Patients with ophthalmoplegia totally recovered.

\section{Discussion}

In patients with host factors for IFI, SFB appears to be frequent in deep sinuses and asymptomatic forms are common. In the presence of clinical and radiological signs suggestive of locoregional invasion, no tissue invasion was detected on histological examination of the sinus mucosa. In all cases, SFB was amenable to minimally invasive surgical treatment, followed by antifungal therapy in complicated cases, resulting in complete cure.

The prevalence of SFB in the general population is unknown. The prevalence of SFB in patients with localized sinusitis operated in our department was $30 \%$. In two retrospective studies on patients undergoing maxillary sinus surgery, the prevalence of SFB was $13 \%$ and $28.5 \%$, respectively ${ }^{(16,17)}$. In a series of 789 cases coded as inflammatory sinus conditions at the Mayo Clinic, including localized, diffuse, operated and nonoperated sinusitis, SFB accounted for $3.7 \%$ of cases ${ }^{(18)}$. SFB is probably underdiagnosed because of its nonspecific symptoms and the presence of asymptomatic forms in $10.5 \%$ of patients in the present study and $11-20 \%$ of cases in the literature $(8,19,20)$.

In patients without host factors for IFI, this study confirmed the usual characteristics of SFB, i.e. predominance of maxillary sites 
Table 3. Comparison of mycological features of sinus fungus balls between patients with or without host factors for invasive fungal infection (group 1 and group 2, respectively).

\begin{tabular}{|c|c|c|c|}
\hline Group (positive cultures) & Group $1(n=6)$ & Group 2 ( $n=31)$ & $\mathbf{p}$ \\
\hline Aspergillus sp & $4(66.7 \%)$ & $26(83.8 \%)$ & ns \\
\hline \multirow[t]{3}{*}{ Other fungal species } & Candida glabrata $(\mathrm{n}=1)$ & Mucor sp $(\mathrm{n}=2)$ & $\mathrm{nr}$ \\
\hline & Schizophyllum commune $(n=1)$ & Candida $\operatorname{sp}(\mathrm{n}=2)$ & $\mathrm{nr}$ \\
\hline & & Clodosporium sp $(\mathrm{n}=1)$ & $\mathrm{nr}$ \\
\hline
\end{tabular}

ns: not significant; nr: not relevant. An unpaired t-test was used for continuous variables and a chi-square test was used for categorical variables. When necessary, Yates' correction or Fisher's exact test were used. A p value $<0.05$ was considered significant.

$(84.6 \%)$ and female predominance ${ }^{(8,13,14,19,20)}$. In the literature, previous dental or endodontic care is reported in $84 \%$ of patients with $\mathrm{SFB}{ }^{(20)}$ suggesting a relationship between maxillary SFB development and dental apex treatment. However, the role of dental canal filling material in the pathogenesis of SFB is still a subject of debate, as SFB can occur in the maxillary sinus in the absence of previous dental care, and may also affect sinuses not anatomically related to the teeth (sphenoidal sinus, ethmoidal sinus, frontal sinus).

Imaging studies are essential for the preoperative diagnosis of SFB, although the radiological signs of SFB can also be observed in other types of chronic sinusitis. CT can be very specific by showing partial or complete heterogeneous sinus opacity associated with an iron-like density with or without microcalcifications. However, SFB may also present as sinus opacity with bone thickening and/or enlargement of the natural ostium ${ }^{(3,4,6)}$. Although MRI can rarely distinguish SFB from sinus mucoecele, it was not performed in uncomplicated cases because of its low specificity and the possibility of artefacts related to the heavy metal (iron and manganese) and calcium content within fungal hyphae. However, in complicated SFB, contrast-enhanced CT and MRI are very useful to evaluate possible meningeal, cerebral and intra-orbital extensions of the infection.

Endoscopic surgery is the gold standard for the treatment of $\operatorname{SFB}(7,13,14,19,21)$. Some surgical teams prefer a video-guided external approach via the anterior wall of the maxillary sinus, as it provides optimal visualization of all of the maxillary sinus and its recesses, preserves ostium physiology, and can be performed under local anesthesia ${ }^{(22)}$. The postoperative recurrence rate is low, zero in this cohort as in other published series ${ }^{(14)}$, and between $4 \%$ and $7 \%$ in other studies ${ }^{(13,18)}$.

In our series of 181 patients with SFB, the prevalence of subjects with host factors for IFI was $10.5 \%$. Gender, age and symptoms of these 19 patients were not different from those of patients without host factors for IFI. Prolonged corticosteroid therapy was reported for four cases. Although the duration of corticosteroid therapy exceeded 24 months, the dosage was always less than $0.3 \mathrm{mg} / \mathrm{kg} /$ day. Interestingly, all diabetic patients had poorly controlled disease indicating the need for further investigation of the role of diabetes mellitus in the pathophysiology of SFB.

In comparison with group 2, SFB in patients with host factors for IFI more frequently involved deep sinuses (ethmoidal, sphenoidal). Four patients of group 1 had complicated SFBs with an acute 'pseudoneoplastic' clinical picture comprising neurological signs and radiological signs of bone lysis in three cases. Several isolated cases of complicated SFB have already been described ${ }^{(9,11,23)}$ with the presence of a host factor for IFI (kidney transplantation with immunosuppressive treatment) in one case ${ }^{(11)}$. Although bone lysis is suggestive of invasive fungal sinus infections ${ }^{(12)}$, fungal tissue invasion was absent in all cases of complicated SFB. The pathophysiology of osteolysis in these cases is unknown, but osteolysis without tissue invasion has already been reported and is thought to be a consequence of chronic inflammation induced by fungal growth ${ }^{(8)}$. Interestingly, three of these four patients were completely asymptomatic before onset of the acute complication. Consequently, the value of systematic CT screening for potentially asymptomatic SFB in patients with host factors for IFI could be evaluated, especially in terms of ethical aspects and costeffectiveness.

All of the patients were successfully operated by endoscopic endonasal surgery. For some authors, osteolysis on CT justifies oral antifungal therapy after surgery ${ }^{(10,23)}$. Based on this recommendation, the three patients of group 1 with osteolysis received antifungal therapy, using azoles in view of their 
fungicidal activity on the fungal species identified and their oral availability. The duration of treatment was empirically at least three months. Precise identification of the fungal species and determination of minimum inhibitory concentrations are essential to subsequently adapt antifungal therapy, especially in complicated forms of SFB. Species identified by culture were mainly Aspergillus sp., as reported in large series of SFB $(8,13,14)$. The detection of yeasts, which are not classically involved in SFB, was considered to be an artefact. Among the other filamentous fungi identified, one case in group 1 was positive for Schizophyllum commune that has sometimes been reported in human diseases and is highly resistant in vitro to amphotericin $B$ and caspofungin ${ }^{(24,25)}$.

The fungal species responsible for SFB were not always identified in this series either because mycological examination was not performed or because mycological examination was performed but the fungus failed to grow on agar medium. In this study, only $32 \%$ of fungal cultures were positive, in line with the literature $(23-79 \% \text { of cases })^{(9,14)}$. The reason for the poor viability of fungal elements in the fungus ball has not been elucidated, but it has been suggested that the various parts of the SFB (i.e. core or outer layer) may have variable growth capacities in culture ${ }^{(26)}$. Moreover, grinding the specimen could further alter its growth and culturing a clump of underground material could be useful. Molecular identification of the species either on cultures or on paraffin-embedded SFB could be a useful tool as a complement to fungal culture, which has a poor sensitivity ${ }^{(24)}$.

\section{Conclusion}

This large series of SFB, although retrospective, shows that host factors for IFI could be present in as many as $10 \%$ of patients. In these patients, SFB more frequently involves deep sinuses and can be complicated by clinical signs of invasion and radiological signs of osteolysis, but with no histological signs of invasion. Endoscopic nasal surgery, combined with antifungal therapy in complicated cases, ensures complete resolution without recurrence. These findings have implications for clinical practice, as CT screening of patients with host factors for IFI could be proposed, as SFB is frequently asymptomatic, especially in this population. A systematic work-up in patients with SFB, especially $\mathrm{HbA} 1 \mathrm{c}$, could also be proposed to detect host factors for IFI.

\section{Acknowledgments}

The authors would like to thank Marie-Christine Leal-Papon for her careful review and useful advice for writing this manuscript and Sandra Asselin for her help in managing the database.

\section{Authorship contribution}

JFP and FB have made substantial contributions to conception and design, data acquisition, and data analysis and interpretation. GT, IAA and VPE were involved in data collection and FZ in statistical analysis. JFP and FB were responsible for drafting the manuscript and critically revising the manuscript for important intellectual content, and have given their final approval of the version to be submitted. All authors have read and approved the final manuscript.

\section{Conflict of interest}

No conflict of interest.

\section{References}

1. Plaignaud M. Observation sur un fungus du sinus maxillaire. J Chirurgie. (Paris) 1791; 1: 111-116.

2. Chakrabarti A, Denning DW, Ferguson BJ, et al. Fungal rhinosinusitis: a categorization and definitional schema addressing current controversies. Laryngoscope. 2009; 119: 1809-1818.

3. Braun JJ and Bourjat P. CT imaging of fungal and nonfungal caseous sinusitis. A report of 50 cases. J Radiol. 2000; 81: 227-231.

4. Khongkhunthian P, Reichart PA. Aspergillosis of the maxillary sinus as a complication of overfilling root canal material into the sinus: report of two cases. J Endod. 2001; 27: 476-478.

5. Krennmair G, Lenglinger F. Maxillary sinus aspergillosis: diagnosis and differentiation of the pathogenesis based on computed tomography densitometry of sinus concre- tions. J Oral Maxillofac Surg. 1995; 53: 657663; discussion 663-654.

6. Legent F, Billet J, Beauvillain C, et al. The role of dental canal fillings in the development of Aspergillus sinusitis. A report of 85 cases. Arch Otorhinolaryngol. 1989; 246: 318-320.

7. Som PM, Dillon WP, Curtin HD, et al. Hypointense paranasal sinus foci: differential diagnosis with MR imaging and relation to CT findings. Radiology. 1990; 176: 777-781.

8. Klossek JM, Serrano E, Peloquin L, et al. Functional endoscopic sinus surgery and 109 mycetomas of paranasal sinuses. Laryngoscope. 1997; 107: 112-117.

9. Ferguson BJ. Fungus balls of the paranasal sinuses. Otolaryngol Clin North Am. 2000; 33: 389-398.

10. Panda NK, Balaji P, Chakrabarti A, et al. Paranasal sinus aspergillosis: its categorization to develop a treatment protocol.
Mycoses. 2004; 47: 277-283.

11. Gungor A, Adusumilli V, Corey JP. Fungal sinusitis: progression of disease in immunosuppression--a case report. Ear Nose Throat. J 1998; 77: 207-210, 215

12. De Pauw B, Walsh TJ, Donnelly JP, et al. Revised definitions of invasive fungal disease from the European Organization for Research and Treatment of Cancer/Invasive Fungal Infections Cooperative Group and the National Institute of Allergy and Infectious Diseases Mycoses Study Group (EORTC/MSG) Consensus Group. Clin Infect Dis. 2008; 46: 1813-1821.

13. Dufour X, Kauffmann-Lacroix C, Ferrie JC, et al. Paranasal sinus fungus ball and surgery: a review of 175 cases. Rhinology. 2005; 43: 34-39.

14. Nicolai P, Lombardi D, Tomenzoli D, et al. Fungus ball of the paranasal sinuses: experience in 160 patients treated with endo- 
scopic surgery. Laryngoscope. 2009; 119 : 2275-2279.

15. deShazo RD, Chapin K and Swain RE. Fungal sinusitis. N Engl J Med. 1997; 337: 254-259.

16. Bambule G, Grigoriu D, Savary $M$, Delacretaz J. Les rhino-sinusites mycotiques et leur traitement. Annales d'Otorhino-laryngologie et chirurgie cervicofaciale. 1982; 99: 531-535.

17. Laskownicka Z, Kurdzielewicz J, Macura A, Okrasińska-Cholewa B. Mycotic sinusitis in children. Mykosen. 1978;21:407-411.

18. Ferreiro JA, Carlson BA, Cody DT, 3rd. Paranasal sinus fungus balls. Head Neck. 1997; 19: 481-486.

19. Serrano E, Percodani J, Flores P, et al. Paranasal sinus aspergilloma. A propos of 45 cases. Ann Otolaryngol Chir Cervicofac. 1996; 113: 86-91.

20. Barry B, Topeza M, Gehanno P. Aspergillosis of the paranasal sinus and environmental factors. Ann Otolaryngol Chir Cervicofac. 2002; 119: 170-173.

21. Grosjean P, Weber R. Fungus balls of the paranasal sinuses: a review. Eur Arch Otorhinolaryngol. 2007; 264: 461-470.
22. Chobillon MA, Jankowski R. What are the advantages of the endoscopic canine fossa approach in treating maxillary sinus aspergillomas? Rhinology 2004; 42: 230235.

23. Rowe-Jones JM, Moore-Gillon V. Destructive noninvasive paranasal sinus aspergillosis: component of a spectrum of disease. J Otolaryngol. 1994; 23: 92-96.

24. Willinger B, Obradovic A, Selitsch B. Detection and identification of fungi from fungus balls of the maxillary sinus by molecular techniques. J Clin Microbiol. 2003; 41: 581-585.

25. Baron O, Cassaing S, Percodani J, et al Nucleotide sequencing for diagnosis of sinusal infection by Schizophyllum commune, an uncommon pathogenic fungus. J Clin Microbiol. 2006; 44: 3042-3043.

26. Dufour X, Kauffmann-Lacroix C, Ferrie JC, et al. Paranasal sinus fungus ball: epidemiology, clinical features and diagnosis. A retrospective analysis of 173 cases from a single medical center in France, 19892002. Med Mycol. 2006; 44: 61-67.
Dr Jean François Papon

Consultation ORL

Hopital H. Mondor

51, avenue du Marechal de Lattre de

Tassigny

94010 Creteil CEDEX

France

Tel: + 33-14-981 2225

Fax: + 33-14-981 2423

E-mail:

jean-francois.papon@hmn.aphp.fr 